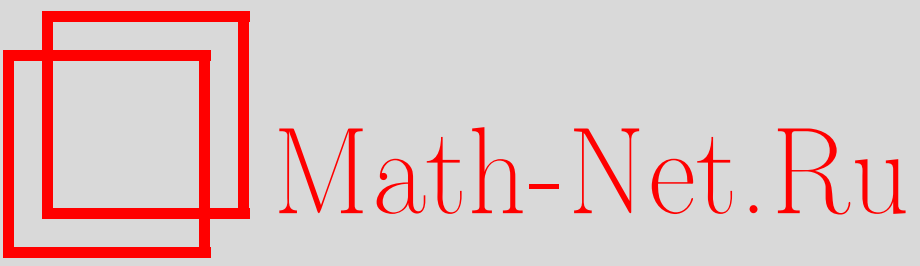

О. В. Моновский, Уравнение движения сферически-симметричной оболочки в релятивистской теории гравитации, ТМФ, 1996, том 107, номер 2, 344-352

DOI: https://doi.org/10.4213/tmf1160

Использование Общероссийского математического портала Math-Net.Ru подразумевает, что вы прочитали и согласны с пользовательским соглашением

http://www.mathnet.ru/rus/agreement

Параметры загрузки:

IP: 52.23 .180 .231

26 апреля 2023 г., 14:44:28 
ТЕОРЕТИЧЕСКАЯ

И МАТЕМАТИЧЕСКАЯ

ФИЗИКА

Том 107, № 2

май, 1996

\title{
О.В. Моновский \\ УРАВНЕНИЕ ДВИЖЕНИЯ \\ СФЕРИЧЕСКИ-СИММЕТРИЧНОЙ ОБОЛОЧКИ В РЕЛЯТИВИСТСКОЙ ТЕОРИИ ГРАВИТАЦИИ
}

\begin{abstract}
В рамках релятивистской теории гравитации получено и проинтегрировано в первом порядке по ньютоновскому потенциалу $U=m / r$ уравнение движения сферически-симметричной дельтаобразной оболочки. Использовались ковариантный закон сохранения энергии-импульса вещества в эффективном римановом пространстве и независимо закон сохранения энергии-импульса для системы вещество + гравитационное поле в пространстве Минковского. Для рассмотренной задачи показана эквивалентность выбранного подхода и классического формализма сингулярных оболочек.
\end{abstract}

Задача о расширяющейся сферически-симметричной оболочке в настояшее время приобретает важное значение в связи с рассмотрением различных космологических инфляционных моделей. В статье [1] в рамках линейного приближения релятивистской теории гравитации (РТГ) было получено решение для гравитационного поля пылевой оболочки конечной толщины. Это решение непрерьвно дифференцируемо во всем пространстве (в том числе и на границах оболочки), ограничено в нуле, убывает на пространственной бесконечности и не содержит падаюших волн, следовательно, согласно теоремам математической физики является единственным.

Чтобы построить приближение данной задачи во втором порядке по ньютоновскому потенциалу $U=m / r$, необходимо найти закон движения оболочки с точностью $m / r$. В данной работе несколькими независимыми способами получено уравнение движения сферически-симметричной дельтаобразной оболочки, в частности, с привлечением введенного Израэлем [2] формализма сингулярных оболочек и приведено его решение.

1. Рассмотрим тензор энергии-импульса сферически-симметричной пылевой дельтаобразной оболочки с законом движения $R(t)$ :

$$
t^{i j}=\frac{1}{4 \pi r^{2}} \delta(r-R(t)) \frac{\rho u^{i} u^{j}}{u^{0}}
$$

(здесь $u^{i}=\frac{d x^{i}}{d s}=\left\{u^{0}, u^{0} v, 0,0\right\}, v=\frac{d R}{d t}=\dot{R}(t)$ - скорость оболочки), и аналогичное выражение для оболочки толшины $a$ :

$$
T^{i j}=\frac{1}{4 \pi r^{2} a}[\theta(r-R(t))-\theta(r-R(t)-a(t))] \frac{\rho u^{i} u^{j}}{u^{0}},
$$


причем граничные условия на скорость вешества $v(t, R(t))=\dot{R}(t)$,

$$
v(t, R(t)+a(t))=\dot{R}(t)+\dot{a}(t), \quad \partial_{r} v(t, R(t))=\partial_{r} v(t, R(t)+a)=0 .
$$

Будем трактовать дельтаобразную оболочку как предел (в интегральном смысле) оболочки конечной толшины, что позволит преодолеть проблемы, возникаюшие при работе с обобщенными функциями, и избежать появления расходимостей:

$$
\lim _{a \rightarrow 0} \int T^{i j} d V=\int t^{i j} d V
$$

где $d V=\sqrt{-\gamma} d^{3} x$ (при переходе к дельтаобразной оболочке справедливо соответствие $\left.\left.\rho(t, r) \rightarrow \rho(t, R(t))\right|_{a=0},\left.u^{i}(t, r) \rightarrow u^{i}(t, R(t))\right|_{a=0}\right)$.

Выберем метрический тензор пространства Минковского в виде $\gamma_{i j}=\operatorname{diag}(1,-1$, $\left.-r^{2},-(r \sin \theta)^{2}\right), \gamma_{i j} \gamma^{j k}=\delta_{i}^{k}$ (ковариантную производную по этой метрике обозначим через $\left.D_{i}\right)$. Докажем, что если функции $\rho(t, r), u^{i}(t, r)$ непрерывны по $r$ и ограничены по $a$ вместе с первыми производными, причем предельный переход $a \rightarrow 0$ сохраняет свойства непрерывности и гладкости, то справедливо равенство

$$
\lim _{a \rightarrow 0} \int D_{i} T^{i j} d V=\int D_{i} t^{i j} d V
$$

Расписываем ковариантные производные с учетом сферической симметрии:

$$
\begin{aligned}
& D_{i} T^{i 0}=\partial_{t} T^{00}+\partial_{r} T^{10}+\frac{2}{r} T^{10} \\
& D_{i} T^{i 1}=\partial_{t} T^{01}+\partial_{r} T^{11}+\frac{2}{r} T^{11}-2 r T^{22} .
\end{aligned}
$$

Подставляя явный вид $T^{i j}$, имеем

$$
\begin{aligned}
& D_{i} T^{i 0}=\frac{1}{4 \pi r^{2} a}\left\{\partial_{t}\left(u^{0} \rho\right)+\partial_{r}\left(u^{0} \rho v\right)-u^{0} \rho \frac{\dot{a}}{a}\right\}[\theta(r-R(t))-\theta(r-R(t)-a(t))]+ \\
& +\frac{1}{4 \pi r^{2} a}\left\{\left(u^{0} \rho v-u^{0} \rho \dot{R}\right)[\delta(r-R(t))-\delta(r-R(t)-a(t))]+u^{0} \rho \dot{a} \delta(r-R(t)-a(t))\right\} .
\end{aligned}
$$

Интегрируем по всему пространству и переходим к пределу $a \rightarrow 0$, принимая во внимание граничные условия на $v$ :

$$
\begin{aligned}
\lim _{a \rightarrow 0} \int D_{i} T^{i 0} d V= & \lim _{a \rightarrow 0} \frac{1}{a} \int_{R(t)}^{R(t)+a}\left[\partial_{t}\left(u^{0} \rho\right)+\partial_{r}\left(u^{0} \rho v\right)-u^{0} \rho \frac{\dot{a}}{a}\right] d r+ \\
& +\lim _{a \rightarrow 0}\left[\left.u^{0} \rho \frac{\dot{a}}{a}\right|_{r=R(t)+a}\right]=\left.\left[\partial_{t}\left(u^{0} \rho\right)+\partial_{r}\left(u^{0} \rho v\right)\right]\right|_{r=R(t), a=0}
\end{aligned}
$$

что совпадает с правой частью (1) при $j=0$ (при вычислении предела для оценки интеграла использовалась теорема о среднем). Аналогично для $j=1$ имеем

$$
\lim _{a \rightarrow 0} \int D_{i} T^{i 1} d V=\left.\left[\partial_{t}\left(u^{0} \rho v\right)+\partial_{r}\left(u^{0} \rho v^{2}\right)\right]\right|_{r=R(t), a=0} .
$$


Доказательство для случая $j=2,3$ тривиально.

В РТГ согласно принципу геометризации движение вешества в эффективном римановом пространстве описывается ковариантным законом сохранения [3]

$$
\nabla_{i} T^{i j}=0
$$

где $\nabla_{i}$ - ковариантная производная по метрике эффективного риманова пространства. Эффективная метрика $g^{i j}$ вводится согласно соотношению $\tilde{g}^{i j}=\sqrt{-g} g^{i j}=\sqrt{-\gamma} \gamma^{i j}$ $\sqrt{-\gamma} \psi^{i j}$, где $g=\operatorname{det} g_{i j}, \gamma=\operatorname{det} \gamma_{i j}, g_{i j} g^{j k}=\delta_{i}^{k}$ (здесь $\psi^{i j}$ - тензор гравитационного поля). Ковариантную производную $\nabla_{i}$ можно тождественно представить в виде

$$
\begin{aligned}
\nabla_{i} T^{i j} & =D_{i} T^{i j}+G_{i l}^{i} T^{l j}+G_{i l}^{j} T^{i l}, \\
G_{j k}^{i} & =\frac{1}{2} \tilde{g}^{i s}\left(D_{j} \tilde{g}_{s k}+D_{k} \tilde{g}_{j s}+D_{s} \tilde{g}_{j k}\right) .
\end{aligned}
$$

Уравнения (2) при $j=2,3$ удовлетворяются автоматически в силу сферической симметрии задачи, движение оболочки определяется из рассмотрения закона сохранения при $j=0,1$. Введем обозначение: $\stackrel{(i)}{f}$-величина $f$ имеет $i$-й порядок по $m$. Выделяя в $(2)$ слагаемые степени малости $O(m / r)$ и $O(m / r)^{2}$ и используя формулу $(3)$, получаем уравнения

$$
\begin{aligned}
& D_{i} \stackrel{(1)}{T}^{i j}=0, \\
& D_{i} \stackrel{(2)}{T}^{i j}=-\stackrel{(1)}{G}_{i l}^{i} \stackrel{(1)}{T}^{l j}-\stackrel{(1)}{G}_{i l}^{j} \stackrel{(1)}{T}^{i l},
\end{aligned}
$$
(1)
(1)
(1)
(1)
(1)

где $\stackrel{G}{j k}_{j k}^{i}=\frac{1}{2} \gamma^{i s}\left(D_{j} \psi_{s k}+D_{k} \psi_{j s}+D_{s} \psi_{j k}\right.$ ) (решение $\psi_{i j}$ для гравитационного поля оболочки конечной толшины получено в [1]).

Решение уравнения (4а) тривиально: $\stackrel{(0)}{v}=$ const, $\stackrel{(1)}{\rho}=$ const, $\stackrel{(0)}{a}=$ const. Пусть $m$ - масса покоя вещества оболочки в первом приближении, определяемая по формуле $u^{0} m=\int t^{00} d V$ (интеграл берется по всему пространству), тогда $\stackrel{(1)}{\rho}=m$.

Раскроем выражение из правой части (4б):

$$
\begin{aligned}
G_{i l}^{i} T^{l 0}+G_{i l}^{0} T^{i l}= & 2 G_{00}^{0} T^{00}+3 G_{01}^{0} T^{01}+G_{11}^{0} T^{11}+ \\
& +G_{01}^{1} T^{00}+G_{11}^{1} T^{01}+2 G_{02}^{2} T^{00}+2 G_{12}^{2} T^{01} \\
G_{i l}^{i} T^{l 1}+G_{i l}^{1} T^{i l}= & G_{00}^{0} T^{01}+G_{01}^{0} T^{11}+G_{00}^{1} T^{00}+ \\
& +3 G_{01}^{1} T^{01}+2 G_{11}^{1} T^{11}+2 G_{02}^{2} T^{01}+2 G_{12}^{2} T^{11} .
\end{aligned}
$$

Так как величины $\stackrel{(1)}{G}_{j k}^{i}$ для дельтаобразной оболочки не являются непрерывными при $r=R(t)$, для получения уравнения движения необходимо совершить полную процедуру интегрального предельного перехода. Используя (1), из (4б) можно получить интегральное соотношение

$$
\int D_{i} \stackrel{(2)}{t}^{i j} d V=-\lim _{a=0} \int\left(\stackrel{(1)}{G}_{i l}^{i} \stackrel{(1)}{T}^{l j}+\stackrel{(1)}{G}_{i l}^{j} \stackrel{(1)}{T}^{i l}\right) d V .
$$


Чтобы вычислить правую часть (5) с требуемой точностью, необходимо иметь значе$\stackrel{(1)}{i}{ }_{j k}$ в области $R(t) \leq r \leq R(t)+a$ (здесь и далее для упрощения выражений величиншы $(0) \quad(0) \quad(0)_{0} \quad(1) \quad(1)$

чины низшего порядка малости $-\stackrel{(0)}{R}(t), \stackrel{(0)}{v}, \stackrel{(0)}{u} 0, \stackrel{(1)}{\rho}, \stackrel{(1)}{\mu}$ и т.д. - будем записывать без индексов). Используя решение из [1], имеем

$$
\begin{aligned}
& \stackrel{(1)}{G}_{00}^{0}=\frac{u^{0} m}{2 a r}\left(1+v^{2}\right)\left[\ln \frac{R_{-}+a}{R_{+}+a}-\ln \frac{1-v}{1+v}\right], \\
& \stackrel{(1)}{G}_{01}^{0}=-\frac{u^{0} m}{2 a r^{2} v}\left(1+v^{2}\right)\left[(R(t)+a) \ln \frac{R_{-}+a}{R_{+}+a}-R(t) \ln \frac{1-v}{1+v}\right] \text {, } \\
& \stackrel{(1)}{G}_{11}^{0}=\frac{u^{0} m}{2 a r^{3} v^{2}}\left\{\left(1+v^{2}\right)\left[(R(t)+a)^{2} \ln \frac{R_{-}+a}{R_{+}+a}-(R(t))^{2} \ln \frac{1-v}{1+v}\right]+\right. \\
& \left.+2 v\left[\left(1+v^{2}\right)(R(t)+a) r-(R(t))^{2}-r^{2} v^{2}\right]\right\}, \\
& \stackrel{(1)}{G_{00}}=\frac{u^{0} m}{2 a r^{2} v}\left(3-v^{2}\right)\left[(R(t)+a) \ln \frac{R_{-}+a}{R_{+}+a}-R(t) \ln \frac{1-v}{1+v}+8 v(r-R(t))\right] \text {, } \\
& \stackrel{(1)}{G_{01}}=-\frac{u^{0} m}{2 a r^{3} v^{2}}\left\{\left(3-v^{2}\right)\left[(R(t)+a)^{2} \ln \frac{R_{-}+a}{R_{+}+a}-(R(t))^{2} \ln \frac{1-v}{1+v}\right]+\right. \\
& \left.+2 v\left[\left(3-v^{2}\right)(R(t)+a) r-3(R(t))^{2}+r^{2} v^{2}\right]\right\} \text {, } \\
& \stackrel{(1)}{G_{11}}=\frac{u^{0} m}{2 a r^{4} v^{3}}\left\{\left(3-v^{2}\right)\left[(R(t)+a)^{3} \ln \frac{R_{-}+a}{R_{+}+a}-(R(t))^{3} \ln \frac{1-v}{1+v}\right]+\right. \\
& \left.+2 v\left[\left(3-v^{2}\right)(R(t)+a)^{2} r-R(t)\left(3(R(t))^{2}-r^{2} v^{2}\right)\right]\right\}, \\
& \stackrel{(1)}{G_{02}^{2}}=\frac{u^{0} m}{2 a r^{3} v^{2}}\left\{\left(3-v^{2}\right)\left[\left(R_{-}+a\right)\left(R_{+}+a\right) \ln \frac{R_{-}+a}{R_{+}+a}-R_{-} R_{+} \ln \frac{1-v}{1+v}\right]+\right. \\
& \left.+2 v\left[\left(3-v^{2}\right)(R(t)+a) r-3(R(t))^{2}+r^{2} v^{2}\right]\right\}, \\
& \stackrel{(1)}{G_{12}^{2}}=-\frac{u^{0} m}{4 a r^{4} v^{3}}\left\{( 3 - v ^ { 2 } ) \left[(R(t)+a)\left(R_{-}+a\right)\left(R_{+}+a\right) \ln \frac{R_{-}+a}{R_{+}+a}-\right.\right. \\
& \left.-R(t) R_{-} R_{+} \ln \frac{1-v}{1+v}\right]+2 v\left[\left(3-v^{2}\right)(R(t)+a)^{2} r-\right. \\
& \left.\left.-R(t)\left(3(R(t))^{2}-r^{2} v^{2}\right)\right]\right\}
\end{aligned}
$$

(здесь $\left.R_{-}=R(t)-v r, R_{+}=R(t)+v r\right)$.

Подставляем выражения (6) в правую часть (5), интегрируем и берем предел $a \rightarrow 0$ :

$$
\begin{aligned}
& -\lim _{a \rightarrow 0} \int\left(\stackrel{(1)}{G}_{i l}^{i} \stackrel{(1)}{T}^{l 0}+\stackrel{(1)}{G}_{i l}^{0} \stackrel{(1)}{T}^{i l}\right) d V=-\frac{\left(u^{0} m\right)^{2}}{2 R^{2}(t)}\left[v\left(3+v^{2}\right)+2\left(1-v^{2}\right) \ln \frac{1-v}{1+v}\right], \\
& -\lim _{a \rightarrow 0} \int\left(\stackrel{(1)}{G}_{i l}^{i} \stackrel{(1)}{T}^{l 1}+\stackrel{(1)}{G}_{i l}^{1} \stackrel{(1)}{T} i l\right) d V=-\frac{\left(u^{0} m\right)^{2}}{2 R^{2}(t)}\left(1-v^{2}\right)\left[1+2 v \ln \frac{1-v}{1+v}\right] .
\end{aligned}
$$


В левой части (5) имеются слагаемые с производными от метрических коэффициентов, входящих в выражение

$$
\stackrel{(1)}{u} 0=-\frac{1}{2}\left(1-v^{2}\right)^{-\frac{3}{2}}\left[\stackrel{(1)}{g}_{00}+2 v \stackrel{(1)}{g}_{01}+v^{2} \stackrel{(1)}{g}_{11}-(\stackrel{(1)}{v})^{2}\right] .
$$

Хотя для решения из [1] при $\left.a \rightarrow 0 \partial_{t} u^{0}\right|_{v=\text { const }}$ и $\left.\partial_{r} u^{0}\right|_{v=\text { const }}$ по отдельности имеют разрыв на границе оболочки, комбинация $\left.\left(\partial_{t}+v \partial_{r}\right) u^{0}\right|_{v=\text { const }}$ непрерывна:

$$
\begin{aligned}
\left.\lim _{r \rightarrow R(t)+0}\left(\partial_{t}+v \partial_{r}\right) u^{0}\right|_{v=\mathrm{const}} & =\left.\lim _{a \rightarrow 0} \frac{1}{a} \int_{R(t)}^{R(t)+a}\left(\partial_{t}+v \partial_{r}\right) u^{0}\right|_{v=\mathrm{const}} d r= \\
& =\left.\lim _{r \rightarrow R(t)-0}\left(\partial_{t}+v \partial_{r}\right) u^{0}\right|_{v=\mathrm{const}}=-\frac{m\left(u^{0}\right)^{4}}{R^{2}(t)} v\left(1+v^{2}\right) .
\end{aligned}
$$

Теперь можно выразить левую часть (5) только через производные от $\stackrel{(1)}{v}$ и $\stackrel{(2)}{\rho}$ :

$$
\begin{aligned}
& \int D_{i}{ }^{(2)}{ }^{i 0} d V=u^{0}\left[\left(u^{0}\right)^{2} \rho v \frac{d \stackrel{(1)}{v}}{d t}+\frac{d^{(2)} \rho}{d t}\right]-\frac{m^{2}\left(u^{0}\right)^{4}}{R^{2}(t)} v\left(1+v^{2}\right), \\
& \int D_{i}{ }^{(2)}{ }^{i 1} d V=u^{0}\left[\left(u^{0}\right)^{2} \rho \frac{d{ }^{(1)}}{d t}+v \frac{d^{(2)}}{d t}\right]-\frac{m^{2}\left(u^{0}\right)^{4}}{R^{2}(t)} v^{2}\left(1+v^{2}\right),
\end{aligned}
$$

где произведена корректная для дельтаобразной оболочки замена $\left(\partial_{t}+v \partial_{r}\right) \rho(t, r) \rightarrow$ $\frac{d}{d t} \rho(t, R(t))$. Итоговая система уравнений на функции $\stackrel{(1)}{v}$ и $\stackrel{(2)}{\rho}$ выглядит следующим образом:

$$
\begin{aligned}
& \left(u^{0}\right)^{2} \rho v \frac{d^{(1)} v}{d t}+\frac{d \stackrel{(2)}{\rho}}{d t}=-\frac{m^{2}\left(u^{0}\right)^{3}}{2 R^{2}(t)}\left[v\left(1-4 v^{2}-v^{4}\right)+2\left(1-v^{2}\right)^{2} \ln \frac{1-v}{1+v}\right] \\
& \left(u^{0}\right)^{2} \rho \frac{d^{(1)}}{d t}+v \frac{d \stackrel{(2)}{\rho}}{d t}=-\frac{m^{2}\left(u^{0}\right)^{3}}{2 R^{2}(t)}\left[1-4 v^{2}-v^{4}+2 v\left(1-v^{2}\right)^{2} \ln \frac{1-v}{1+v}\right] .
\end{aligned}
$$

Решая ее относительно $\frac{d_{v}^{(1)}}{d t}$ и $\frac{d_{\rho}^{(2)}}{d t}$, имеем

$$
\begin{aligned}
& \frac{d \stackrel{(1)}{v}}{d t}=-\frac{m u^{0}}{2 R^{2}(t)}\left(1-4 v^{2}-v^{4}\right) \\
& \frac{d^{(2)}}{d t}=-\frac{m^{2}}{R^{2}(t)} \sqrt{1-v^{2}} \ln \frac{1-v}{1+v} .
\end{aligned}
$$

Заметим, что выражение $\left(\partial_{t}+v \partial_{r}\right) \stackrel{(1)}{\gamma / g}$ на решении из [1] непрерьвно по $r$. Прямые вычисления дают

$$
\left.\left(\partial_{t}+v \partial_{r}\right) \stackrel{(1)}{\gamma / g}\right|_{r=R(t)}=-\frac{m}{R^{2}(t)} \sqrt{1-v^{2}} \ln \frac{1-v}{1+v} .
$$

Сравнивая результат (11) с (10б), мы можем с требуемой точностью записать $\stackrel{(2)}{\rho}=$ $m \sqrt{\gamma / g}$. Величина $\mu=\frac{1}{4 \pi r^{2}} m \delta(r-R(t))$ является сохраняющейся в данном приближении плотностью массы: $\partial_{t} \mu+\partial_{r}(v \mu)=O\left((m / r)^{3}\right)$ (здесь величины $v(t)$ и $R(t)$ представлены с точностью до $m$ ). 
2. Уравнения РТГ можно записать в виде

$$
\begin{aligned}
\tilde{g}^{p q} D_{p} D_{q} g^{i j} & =16 \pi\left(\widetilde{\widetilde{T}}^{i j}+\tilde{\tilde{\tau}}^{i j}\right), \\
D_{i} \tilde{g}^{i j} & =0
\end{aligned}
$$

где в правой части стоят тензоры энергии-импульса вещества и гравитационного поля веса +2 :

$$
\begin{gathered}
\tilde{\widetilde{T}}^{i j}=(-g) T^{i j} \\
\tilde{\tilde{\tau}}^{i j}=\frac{1}{32 \pi}\left[\tilde{g}^{i j}\left(\tilde{g}_{m n} D_{p} \tilde{g}^{m q} D_{q} \tilde{g}^{n p}+\frac{1}{2} \tilde{g}^{m n} D_{m} \tilde{g}_{p q} D_{n} \tilde{g}^{p q}+\frac{1}{4} \tilde{g}_{m n} \tilde{g}_{p q} \tilde{g}^{r s} D_{r} \tilde{g}^{m n} \tilde{g}^{p q}\right)-\right. \\
-2 \tilde{g}_{m n} \tilde{g}^{j s} D_{p} \tilde{g}^{i m} D_{s} \tilde{g}^{p n}-2 \tilde{g}_{m n} \tilde{g}^{i s} D_{p} \tilde{g}^{j m} D_{s} \tilde{g}^{p n}+2 \tilde{g}_{m n} \tilde{g}^{p q} D_{p} \tilde{g}^{i m} D_{q} \tilde{g}^{j n}- \\
\left.-\frac{1}{2} \tilde{g}_{m n} \tilde{g}_{p q} \tilde{g}^{i s} \tilde{g}^{j r} D_{s} \tilde{g}^{m n} D_{r} \tilde{g}^{p q}-\tilde{g}^{j n} \tilde{g}^{i m} D_{m} \tilde{g}_{p q} D_{n} \tilde{g}^{p q}+2 D_{p} \tilde{g}^{i m} D_{m} \tilde{g}^{p j}\right] .
\end{gathered}
$$

Выделим уравнения на поле $\psi^{i j}$ во втором приближении по $m / r$ :

$$
\begin{aligned}
\gamma^{p q} D_{p} D_{q} \stackrel{(2)}{\psi}^{i j} & =-16 \pi\left(\stackrel{(2)}{\mathcal{T}}^{i j}+\stackrel{(2)}{\tau}^{i j}\right), \\
D_{i}{ }^{(2)} & i j=0
\end{aligned}
$$

здесь $\mathcal{T}^{i j}=g / \gamma T^{i j}, \stackrel{(2)}{\tau}{ }^{i j}$ - часть тензора энергии-импульса, учитывающая вклад чисто гравитационного поля первого приближения $\stackrel{(1)}{\psi}, i j$ :

$$
\begin{aligned}
& \stackrel{(2)}{\tau}_{i j}=\frac{1}{32 \pi}\left[\gamma ^ { i j } \left(\gamma_{m n} D_{p} \stackrel{(1)}{\psi}{ }^{m q} D_{q} \stackrel{(1)}{\psi}^{n p}-\frac{1}{2} \gamma^{m n} D_{m} \stackrel{(1)}{\psi}_{p q} D_{n} \stackrel{(1)}{\psi}^{p q}+\right.\right. \\
& \left.+\frac{1}{4} \gamma_{m n} \gamma_{p q} \gamma^{r s} D_{r} \stackrel{(1)}{\psi}^{m n} D_{s} \stackrel{(1)}{\psi} p q\right)-2 \gamma_{m n} \gamma^{j s} D_{p}{ }^{(1)} i m D_{s}{ }^{(1)} p n \\
& -2 \gamma_{m n} \gamma^{i s} D_{p} \stackrel{(1)}{\psi}^{j m} D_{s} \stackrel{(1)}{\psi}^{p n}+2 \gamma_{m n} \gamma^{p q} D_{p}{ }^{(1)}{ }^{i m} D_{q}{ }^{(1)}{ }^{j n}- \\
& -\frac{1}{2} \gamma_{m n} \gamma_{p q} \gamma^{i s} \gamma^{j r} D_{s} \stackrel{(1)}{\psi}^{m n} D_{r} \stackrel{(1)}{\psi}^{p q}+\gamma^{j n} \gamma^{i m} D_{m} \stackrel{(1)}{\psi}_{p q} D_{n} \stackrel{(1)}{\psi}^{p q}+ \\
& \left.+2 D_{p}{ }^{(1)}{ }^{i m} D_{m}{ }^{(1)}{ }^{p j}-2 \stackrel{(1)}{\psi}^{p q} D_{p} D_{q}{ }^{(1)}{ }^{i j}\right] \text {. }
\end{aligned}
$$

Следствием (12) является дифференциальньй закон сохранения

$$
D_{i}\left(\stackrel{(2)}{\mathcal{T}}^{i j}+\stackrel{(2)}{\tau}^{i j}\right)=0
$$

Для $\mathcal{T}^{i j}$ справедливо аналогичное (1) тождество

$$
\lim _{a \rightarrow 0} \int D_{i} \mathcal{T}^{i j} d V=\int D_{i}\left(g / \gamma t^{i j}\right) d V
$$


Используя его, можно переписать (13) в интегральном виде:

$$
\int D_{i}\left(g / \stackrel{(2)}{\gamma} t^{i j}\right) d V=-\lim _{a \rightarrow 0} \int D_{i} \stackrel{(2)}{\tau} i j d V
$$

Вычисляя правую часть (14) для решения из [1], после предельного перехода $a \rightarrow 0$ получаем (отличный от нуля вклад дает только область $R(t) \leq r \leq R(t)+a)$ :

$$
\begin{aligned}
& -\lim _{a \rightarrow 0} \int D_{i}{ }^{(2)} \tau^{i 0} d V=-\frac{\left(u^{0} m\right)^{2}}{2 R^{2}(t)}\left[v\left(3+v^{2}\right)-2\left(1-v^{2}\right) \ln \frac{1-v}{1+v}\right] \\
& -\lim _{a \rightarrow 0} \int D_{i}{ }^{(2)} \tau^{i} d V=-\frac{\left(u^{0} m\right)^{2}}{2 R^{2}(t)}\left(1-v^{2}\right)\left[1-2 v \ln \frac{1-v}{1+v}\right] .
\end{aligned}
$$

Раскрываем левую часть (14) при $j=0,1$, применяя равенство (11):

$$
\int D_{i}\left(g / \stackrel{(2)}{\gamma} t^{i j}\right) d V=\int D_{i} t^{(2)} i j d V+\frac{2 m^{2}}{R^{2}(t)} v^{i} v^{j} \ln \frac{1-v}{1+v} .
$$

С учетом этого результата и соотношения (8) закон сохранения (14) приводит для $\stackrel{(1)}{v}$ и $\stackrel{(2)}{\rho}$ к системе $(9)$.

3. В четырехмерном пространстве-времени дельтаобразная оболочка описывается гиперповерхностью $\Sigma: r=R(t)$. Полная производная по времени от векторной функции $A^{i}$, заданной на этой поверхности, определяется формулой

$$
\frac{\delta A^{i}}{\delta t}=\frac{\partial A^{i}}{\partial t}+A^{j} \Gamma_{j k}^{i} \frac{d x^{k}}{d t}
$$

Введем на $\Sigma$ вектор нормали $n^{i}=\left\{-\stackrel{\circ}{R}, u^{0}, 0,0\right\}$ (мы обозначили $\stackrel{\circ}{R}=\frac{d R}{d s}$ ), четырехскорость $u^{i}=\left\{u^{0}, \stackrel{\circ}{R}, 0,0\right\}$ является касательной к $\Sigma$. Тогда, как показал Израэль [2], уравнение движения оболочки запишется в виде

$$
\left.n_{i} \frac{\delta u^{i}}{\delta s}\right|_{r=R(t)-0}+\left.n_{i} \frac{\delta u^{i}}{\delta s}\right|_{r=R(t)+0}=0 .
$$

Чтобы исключить $\frac{\delta^{2} t}{\delta s^{2}}$ из выражения $n_{i} \frac{\delta u^{i}}{\delta s}=-\stackrel{\circ}{R} \frac{\delta^{2} t}{\delta s^{2}}+u^{0} \frac{\delta^{2} R}{\delta s^{2}}$, продифференщируем тождество $u_{i} u^{i}=1$ :

$$
0=u_{i} \frac{\delta u^{i}}{\delta s}=\frac{\delta^{2} t}{\delta s^{2}}\left(g_{00} u^{0}+g_{01} \stackrel{\circ}{R}\right)+\frac{\delta^{2} R}{\delta s^{2}}\left(g_{01} u^{0}+g_{11} \stackrel{\circ}{R}\right) .
$$

Используя $g_{00}\left(u^{0}\right)^{2}+2 g_{01} \stackrel{\circ}{R} u^{0}+g_{11} \stackrel{\circ}{R}{ }^{2}=1$, окончательно имеем

$$
n_{i} \frac{\delta u^{i}}{\delta s}=\frac{\delta^{2} R}{\delta s^{2}}\left(g_{00} u^{0}+g_{01} \stackrel{\circ}{R}\right)^{-1}
$$


Раскрываем полную производную:

$$
\frac{\delta^{2} R}{\delta s^{2}}=\frac{d^{2} R}{d s^{2}}+\Gamma_{i j}^{1} u^{i} u^{j}=\frac{d^{2} R}{d s^{2}}+\Gamma_{00}^{1}\left(u^{0}\right)^{2}+2 \Gamma_{01}^{1} \stackrel{\circ}{R} u^{0}+\Gamma_{11}^{1} \stackrel{\circ}{R^{2}}
$$

(здесь $\Gamma_{i j}^{k}$ - символы Кристоффеля по метрике $g_{i j}$ ).

Подставляя $(16),(17)$ в (15) и удерживая только члены порядка $m$, получаем искомое уравнение движения

$$
\frac{d^{2} R}{d s^{2}}=\frac{1}{2}\left[\left.\left.\stackrel{(1)}{\Gamma}\right|_{i j} ^{1}\right|_{r=R(t)-0}+\left.\stackrel{(1)}{\Gamma}_{i j}^{1}\right|_{r=R(t)+0}\right] \stackrel{(0)}{u} i \stackrel{(0)}{u} j
$$

Нам потребуются значения следующих символов Кристоффеля для решения из [1]: снаружи оболочки $r>R(t)-$

$$
\begin{aligned}
& \stackrel{(1)}{\Gamma}{ }_{00}=\frac{u^{0} m}{2 r^{2} v}\left[\left(3-v^{2}\right) \ln \frac{1-v}{1+v}+8 v\right], \\
& \stackrel{(1)}{\Gamma_{01}}=-\frac{u^{0} m}{r^{3} v^{2}}\left[\left(3-v^{2}\right) \ln \frac{1-v}{1+v}+6 v\right] R(t), \\
& \stackrel{(1)}{\Gamma}{ }_{11}^{1}=\frac{u^{0} m}{2 r^{4} v^{3}}\left\{3\left(3-v^{2}\right)(R(t))^{2} \ln \frac{1-v}{1+v}+2 v\left[9(R(t))^{2}-r^{2} v^{2}\right]\right\} ;
\end{aligned}
$$

в полости оболочки $r<R(t)-$

$$
\begin{aligned}
& \stackrel{(1)}{\Gamma}{ }_{00}^{1}=\frac{u^{0} m}{2 r^{2} v}\left(3-v^{2}\right)\left[\ln \frac{R_{-}}{R_{+}}+2 r v \frac{R(t)}{R_{+} R_{-}}\right], \\
& \stackrel{(1)}{\Gamma_{01}^{1}}=-\frac{u^{0} m}{r^{3} v^{2}}\left(3-v^{2}\right)\left\{R(t) \ln \frac{R_{-}}{R_{+}}+r v\left[1+\frac{(R(t))^{2}}{R_{+} R_{-}}\right]\right\}, \\
& \stackrel{(1)}{\Gamma}{ }_{11}^{1}=\frac{u^{0} m}{2 r^{4} v^{3}}\left(3-v^{2}\right)\left\{3 R(t) \ln \frac{R_{-}}{R_{+}}+2 r v\left[2+\frac{(R(t))^{2}}{R_{+} R_{-}}\right]\right\} R(t)
\end{aligned}
$$

(здесь действует прежнее соглашение относительно обозначения величин низшего порядка по $m$ ).

Прямые вычисления дают, что решению из [1] соответствует следуюшее уравнение движения оболочки:

$$
\frac{d^{2} R}{d s^{2}}=-\frac{u^{0} m}{2 R^{2}}
$$

Переходим к дифференцированию по времени:

$$
\frac{d^{2} R}{d s^{2}}=\left(u^{0}\right)^{2} \frac{d^{2} R}{d t^{2}}+u^{0} \frac{d u^{0}}{d t} \frac{d R}{d t} .
$$

Преобразования, аналогичные использованным при переходе от системы (7) к системе (9), приводят к результату

$$
\left.\frac{d^{(1)} u^{0}}{d t}\right|_{r=R(t)}=-\frac{m\left(u^{0}\right)^{4}}{R^{2}(t)} v\left(1+v^{2}\right)+\left(u^{0}\right)^{3} v \frac{d^{(1)} v}{d t} .
$$


Полученное уравнение на функцию $\stackrel{(1)}{v}$ совпадает с (10a):

$$
\frac{d \stackrel{(1)}{v}}{d t}=-\frac{m u^{0}}{2 R^{2}(t)}\left(1-4 v^{2}-v^{4}\right)
$$

Можно заключить, что для нашей задачи прямое использование законов сохранения для получения уравнения движения оболочки эквивалентно классическому формализму сингулярных оболочек.

Интегрирование уравнения движения с начальными условиями $R(0)=R_{0}, \frac{d R(0)}{d t}=v$ дает закон движения оболочки

$$
R(t)=R_{0}+v t+\frac{m u^{0}}{2 v^{2}}\left(1-4 v^{2}-v^{4}\right)\left[\ln \frac{R_{0}+v t}{R_{0}}-\frac{v t}{R_{0}}\right] .
$$

\section{Список литературы}

[1] Власов А. А., Моновский О. В. // ТМФ. 1992. Т. 91. № 2. С. 334-345.

[2] Israel $W$. // Nuovo Cim. 1966. V. B44. № 1. P. 1-14.

[3] Логунов А.А., Мествиришвили М. А. Релятивистская теория гравитации. М.: Наука, 1989.

Московский государственный

Поступила в редакцию

университет

3.V.1995 г.

\section{THE EQUATION OF MOTION OF THE SPHERICALLY SYMMETRIC SHELL IN RELATIVISTIC GRAVITATIONAL THEORY}

The equation of motion of the singular spherically symmetric shell is found and integrated with accuracy to the first order of the Newton potential $U=m / r$. The energy-momentum conservation laws, one for matter in effective curved space-time and the other for both matter and gravitational field in Minkowski space-time are used independently. These methods appear to be equivalent to the classical formalism of singular hypersyrfaces suggested. 